\title{
Viral tunes: changes in musical behaviours and interest in coronamusic predict socio-emotional coping during COVID-19 lockdown
}

\author{
Lauren K. Fink (10 1,2,8凶 , Lindsay A. Warrenburg (1) ${ }^{3,8 凶}$, Claire Howlin (1) ${ }^{4}$, William M. Randall ${ }^{5}$, \\ Niels Chr. Hansen (D) ${ }^{6,7,9}$ \& Melanie Wald-Fuhrmann (1) 1,2,9
}

Beyond immediate health risks, the COVID-19 pandemic poses a variety of stressors, which may require expensive or unavailable strategies during a pandemic (e.g., therapy, socialising). Here, we asked whether musical engagement is an effective strategy for socio-emotional coping. During the first lockdown period (April-May 2020), we surveyed changes in music listening and making behaviours of over 5000 people, with representative samples from three continents. More than half of respondents reported engaging with music to cope. People experiencing increased negative emotions used music for solitary emotional regulation, whereas people experiencing increased positive emotions used music as a proxy for social interaction. Light gradient-boosted regressor models were used to identify the most important predictors of an individual's use of music to cope, the foremost of which was, intriguingly, their interest in "coronamusic." Overall, our results emphasise the importance of real-time musical responses to societal crises, as well as individually tailored adaptations in musical behaviours to meet socio-emotional needs.

\footnotetext{
${ }^{1}$ Department of Music, Max Planck Institute for Empirical Aesthetics, Frankfurt am Main, Germany. ${ }^{2}$ Max Planck NYU Center for Language, Music and Emotion (CLaME), Frankfurt am Main, Germany. ${ }^{3}$ Sonde Health, Inc, Boston, MA, USA. ${ }^{4}$ School of Psychology, Queen Mary University of London, London, UK. ${ }^{5}$ Department of Music, Art and Culture Studies, University of Jyväskylä, Jyväskylä, Finland. ${ }^{6}$ Aarhus Institute of Advanced Studies, Aarhus University, Aarhus, Denmark. ${ }^{7}$ Center for Music in the Brain, Aarhus University \& Royal Academy of Music Aarhus/Aalborg, Aarhus, Denmark. ${ }^{8}$ These authors contributed equally: Lauren K. Fink, Lindsay A. Warrenburg. ${ }^{9}$ These authors jointly supervised this work: Niels Chr. Hansen, Melanie Wald-Fuhrmann.凶email: lauren.fink@ae.mpg.de; lindsay.a.warrenburg@gmail.com
} 


\section{Introduction}

pidemics have been recurring events throughout human history (Hays, 2005) and evolution (Prohaska et al., 2019). As globalisation increases the risk that outbreaks develop into pandemics, effective containment measures are crucial. While reducing public health risk, these measures may create massive economic, societal, and political co-crises which deeply affect socio-emotional wellbeing. Such is the case during the COVID-19 pandemic. In addition to fear of the virus (Lippold et al., 2020) and elevated stress levels due to physical-distancing measures (Best et al., 2020), many people faced (and continue to face) reduced income or unemployment, the need to combine working from home with childcare duties, uncertainty about the future, and social isolation. Evidence is accumulating for severe negative mental health impacts, such as increased levels of anxiety, depression, stress, loneliness, and impaired sleep quality (Dagnino et al., 2020; Dawel et al., 2020; Franceschini et al., 2020; Groarke et al., 2020; Hur et al., 2020; Lenzo et al., 2020; Lippold et al., 2020; Parlapani et al., 2020; Rajkumar, 2020; Rodríguez-Rey et al., 2020; Rossi et al., 2020).

People's ability to maintain mental wellbeing affects their willingness to comply with necessary containment regulations (Jetten et al., 2020) and helps prevent social unrest, stigma, and violence (Barrett, 2020; Polo, 2020; Villa et al., 2020). Behavioural data can illuminate which coping strategies effectively strengthen individual and community resilience (Betsch, 2020; Kroska et al., 2020). Here, we investigate one particularly widespread, accessible, and lockdown-compatible coping behaviour: engaging with music. While a variety of coping strategies gained prominence during the COVID-19 pandemic, none received as much broad public support and media endorsement as music. Videos of Italians singing from balconies swiftly multiplied and diversified into multifaceted reports of new ways of engaging with music during lockdown. In Asia, a music video commissioned by the Vietnamese government encouraging proper hand-washing technique and social distancing precautions went viral and became the impetus for a TikTok dance challenge ${ }^{1}$. Quickly, shared narratives arose praising music for providing social connection and comfort, despite physical distancing, as well as positivity and humour against an all-too-dominant backdrop of negative emotions (Langley and Coutts, 2020; Macdonald, 2020; Taylor, 2020).

The outburst of musical creativity was multifarious and resulted in a set of dedicated repertoires and practices that refer to, or are inspired by, the pandemic and can be termed "coronamusic." Hansen et al. (2021) broadly define coronamusic as the audiovisual products resulting from any number of diverse forms of musical engagement (listening, playing, dancing, composing, rehearsing, improvising, discussing, exploring, innovating) which explicitly or implicitly reference the coronavirus and/ or life during the pandemic. As such, coronamusic is not a particular genre produced by a certain practice, but rather a collection of cultural products which share a common theme or inspiration. For examples of coronamusic from around the globe, please see: https://osf.io/y7z28/ (which can be sorted by country of interest). In brief, people composed new pieces, created themed playlists, and reworked famous songs, drawing new meanings from an unfamiliar context. Professional and lay music ensembles experimented with playing together via videoconferencing software, and artists streamed performances from their homes with tens of thousands of viewers watching as a substitute for live music. Virtual concert activity peaked in two multi-hour, all-star shows that were live-streamed on YouTube to millions of viewers: the "One World: Together at Home" event under the aegis of Lady Gaga on April 18 and the "One Love Asia Concert" on May $27 .^{2}$ These concerts were created to provide emotional support, call for considerate behaviour, and raise funds for the $\mathrm{WHO}$, UNICEF, and local charities-all through music.

The public narrative of music's benefits aligns with scientific studies on the socio-emotional coping potential of music in everyday life (DeNora, 2000; North and Hargreaves, 2008; Juslin and Sloboda, 2010), as well as with historical evidence from earlier pandemics (Chiu, 2020). Making and listening to music are potent means of emotional self-regulation (Saarikallio, 2011; Trehub, 2003). Specifically, musical behaviours provide effective strategies for reducing anxiety (Cuypers et al., 2012; Theorell and Kreutz, 2012; Tarr et al., 2014), depression (Cuypers et al., 2012; Theorell and Kreutz, 2012; Aalbers et al., 2017), and stress (de Witte et al., 2020). Music has additionally been shown to serve as a social surrogate by providing a sense of belonging (Derrick et al., 2009; Gabriel and Young, 2011) and making people feel like they have company (Schäfer and Eerola, 2020; Schäfer et al., 2020).

It is unknown, however, whether the effectiveness of musical engagement as a socio-emotional coping strategy generalises to a stressor with such a broad and deep collective impact as the COVID-19 pandemic. It is also unclear what role dedicated repertoires and practices might play in these circumstances. Additionally, there are situation-specific factors that might affect the degree to which people use music to cope, including access to music (versus other potential coping resources during lockdown) and the perceived severity of the pandemic and its consequences. Individual variables influencing musical engagement more generally, such as musical attitudes and behaviours (Schäfer, 2016), psychological traits (Garrido et al., 2015; Ladinig et al., 2019; Howlin and Rooney, 2021; Warrenburg, 2020), and demographics, may also be relevant. The relative importance of these factors in predicting how helpful music is for coping under such unprecedented circumstances remains to be determined.

This large scale $(N>5000)$, cross-cultural, broadly representative study tests whether two fundamental musical behaviours-listening to and making music-provided successful socio-emotional coping strategies in the context of the initial COVID-19 lockdown in early 2020. Building on theories about the dependence of music selection behaviour on social situations and functional goals (Schäfer and Sedlmeier, 2009; Greb et al., 2018), we establish the degree to which changes in musical engagement were associated with coping, and we identify the most important predictors from a set of variables much broader and more diverse than used in previous studies. Data were collected from demographically representative samples (in terms of age, gender, and education) in six countries (France, Germany, India, Italy, UK, USA; $N=5113$; age: $M=44$ years, $\mathrm{SD}=16$; gender: $53.8 \%$ women; education $M=15$ years, $\mathrm{SD}=6$; see supplemental material, Appendix 1, for detailed demographics).

\section{Methods}

Questionnaire development. A custom questionnaire was developed to explore changes in musical engagement during the COVID-19 pandemic and lockdown. The questionnaire was jointly developed by all authors in English. Translations into German, French, and Italian were each prepared by two native speakers with either a professional background in translating (French and Italian version) or in questionnaire design (German version). The final questionnaires in each language can be accessed in a dedicated GitHub repository (https://github.com/ lkfink/CMQ). The survey consisted of seven core sections that measured: (1) basic demographic information; (2) living situation, employment, and importance of various leisure activities and chores during lockdown; (3) changes in listening formats 
(Braun and Krause, 2020; e.g., streaming services), listening situations (Greb et al., 2018; e.g., while doing housework), forms of making music, and music selection (e.g., nostalgic music, diverse music) during lockdown; (4) whether or not people experienced making music as helping them cope emotionally with the lockdown situation, feel connected to others, and serve as a replacement for social interaction as well as changes in the importance of functions that making music served during lockdown, based on previously developed scales (Schäfer and Sedlmeier, 2009; Groarke and Hogan, 2016, 2018; Randall and Rickard, 2017); (5) similar questions for music listening; (6) open-ended questions about changes in musical behaviour and specific repertoire listened to or played (not reported here); as well as (7) various questions about musicianship (OMSI, Ollen, 2006), importance of music, musical participation, personality traits (BFI-10, Rammstedt and John, 2007), and self-reported changes in health and wellbeing due to the pandemic. Sections 3-5 of the questionnaire were only shown to participants who reported listening to or making music in section 2 .

For most items on the survey, we were assessing changes in the importance, frequency or likelihood of a behaviour in relation to before lockdown measures were introduced. For example, "Since corona crisis measures were introduced in your area, do you now listen to more or less music from your childhood and/or adolescence?" Participants responded to such questions using a 7 -point Likert scale that ranged from 1 "significantly less" to 4 "about the same" to 7 "significantly more." For some items, such as the changes in functions of music listening/making music, we also included answer options for "never do this" and "prefer not to say."

Data collection and participants. Data were collected from participants experiencing lockdown measures during the first wave of the COVID-19 pandemic in mid-April through mid-May 2020 in France, Germany, India, Italy, USA (New York state only), and the UK. These countries were selected because they differed in terms of the urgency of the pandemic at that time (number of cases and deaths), the severity of lockdown measures, the types and degree of publicly acknowledged corona-related musical behaviours, but also culturally. Online data collection services Prolific (for the UK sample) and IPSOS-MORI (all other samples) were used to recruit participants from the general population, providing sample sizes of 700 to 1000 from each country. Country-specific quotas were set regarding gender, age, and education levels so as to aim for a broadly representative sample along these three dimensions. This was not entirely possible for India, however, because taking the survey required participants to be literate in English, which is not the case for a large part of the population. As a consequence, the Indian sample cannot claim to be representative for the entire Indian population but rather only the smaller subpopulation of those who speak English who typically have higher levels of education and income than the average population. All responses were collected online using Qualtrics. Participants could pause the survey and return at a later time. The median survey completion time was $17 \mathrm{~min}$.

Participants provided informed written consent and could cease completion at any time. They were screened out of the survey if they reported an age under 18, or if they were part of a gender, age, or education level group whose quota had already been met. A research ethics exemption was obtained from University College Dublin Human Research Ethics Committee (REERN: HS-E-20-62). After cleaning the data using the preprocessing procedures outlined below, our sample had the demographic distributions shown in Supplementary Material, Appendix 1. Information about COVID-19 deaths and the strictness of governmental measures at the time of data collection can also be found in Supplementary Appendix 1.

Data preprocessing. All data were analysed in Python 3 (Python Software Foundation, https://www.python.org/) using custom scripts and freely available packages. All figures and models can be recreated using the code and data table provided in our Github repository (https://github.com/lkfink/CMQ).

Participants falling into the lowest 10th percentile of survey taking duration (based on the sections of the survey they completed) were removed from further analyses. We also removed participants who exhibited no range down any of the large matrix questions related to activities, music listening functions, or making music functions, so long as their answer was not "no change", "never do this", or "prefer not to say", which were regarded as potentially meaningful answers in these contexts. Categorical questions and questions with options to select multiple answers, like "How is your work situation affected by the coronavirus crisis?" were one-hot encoded prior to analysis (i.e., a binary column was created for each possible response item, with a 0 or 1 indicating whether participants selected that answer item). Finally, people who responded to a question with the answer "I never do this" were recoded as a "4" ("no change before vs. during the pandemic").

To analyse participants' responses as a function of governmental actions and COVID-19 cases in their respective countries at the date of survey taking, we sourced data from the Oxford COVID-19 Government Response Tracker (Hale et al., 2021). For each participant, we included all Oxford data from their surveytaking date. Oxford measures of interest included the cumulative number of confirmed COVID-19 cases and deaths, as well as the measures devised by the Oxford group: "Containment Health Index", "Economic Support Index", "Government Response Index", and "Stringency Index". Confirmed deaths and the stringency index in each country, across our data collection periods in each country, are plotted in Supplementary Material, Fig. A1. All Oxford variables were included in our statistical models, with the hypothesis that the urgency of the pandemic and severity of lockdown might impact participants' engagement with music as a means of coping (see section below).

Rankings. On the country level, we report rankings based on means, rather than raw means, so as to avoid any differences in response bias between countries, which seemed to exist primarily in the Indian sample in the form of acquiescence bias, which has also been found for this population in earlier research (Harzing, 2006). For each matrix variable of interest (e.g., music listening functions), in each country, we excluded "prefer not to say" answers. We then subtracted 4 from each answer (because we used a 7 -point scale, centred on 4 ), to centre the data around 0 , with negative numbers representing a decrease in importance, 0 no change, and positive numbers, an increase in importance. We then calculated the mean for each item and ranked all items based on their mean, within each country. If a tie between items occurred, the items were assigned the average rank of all items in question. For visualisation purposes, we plot the rank of each item, for each country, sorted by the median rank of each item across all countries. A low rank (i.e., 1) indicates that that item changed most in importance, compared to before the onset of the coronavirus crisis, and vice versa. As participants may have chosen to leave some questions blank or preferred not to answer, the final sample size for each analysis is variable, depending upon the number of missing answers for that question. This number is therefore reported alongside all figures. 
Factor analysis. To assess individual differences with respect to musical engagement, an exploratory factor analysis (EFA) was conducted containing the personality and demographic data. Missing values were imputed with the variable median with regard to the participant's country. Namely, if a person residing in India had a missing value for loneliness, the imputed value was the median score for loneliness for participants residing in India. As the median score for all of the variables was almost always 4 ("no change from before coronavirus to during coronavirus"), this approach is conservative in nature and minimises the possibility that the factor analysis will be influenced by outliers.

The 51 final variables used in the factor analysis included information about the person's living situation (e.g., whether their location during the pandemic was the place they call home, if they were living in a rural/suburban/urban area, if they were living alone or with a partner or with children, the number of times they left the house per week), their employment status before the pandemic (e.g., employed full time, employed part time, homemaker or caregiver), the ways their work was affected by the pandemic (e.g., if they lost their job, if they began working from home, if they were homeschooling their children), their contact with the coronavirus (e.g., they are infected, they live with someone infected, they have professional contact with someone infected), their demographics (e.g., age, years of education, gender), changes in their health and wellbeing during the pandemic (e.g., levels of loneliness, stress, positive emotions), and personality characteristics (e.g., extraversion, openness). None of the pairs of variables showed a correlation greater than 0.7 , suggesting that the correlations among variables would not bias the factor analysis results. Finally, the continuous columns were scaled with regard to the person's country of residence, in order to minimise any potential response bias differences between countries. "Minmax" scaling was used, so that all values were scaled to a given range (the minimum and maximum value for that variable in that country).

Two preliminary tests were conducted to examine whether the correlation matrix of the variables contained redundancies that could be explained through latent variables. The results of Bartlett's test of sphericity are consistent with the notion that latent factors can explain correlations in the selected variables (statistic $=67678.790, p<0.001$ ). Similarly, the results of the Kaiser-Meyer-Olkin test show that the correlation matrix is not an identity matrix and the variance among individual characteristics could be explained with latent factors (KMO statistic $=0.548$ ). The results of a scree plot (of the eigenvalues) suggested a solution with around six factors. Therefore, the EFA was conducted with six factors and a quartimax rotation.

Factor 1 was labelled Negative Emotions, as it was best explained by all the negative emotion variables polled (depression, stress, anxiety, loneliness, negative emotions). Factor 2 was labelled Age, as the highest loadings were a person's age, if they lived with a parent, were a student, and if they were retired. Factor 3 was labelled Positive Emotions, as it was best explained with positive emotions, energetic arousal, and general health. The highest variable loadings on Factor 4 were all related to a person's Living Situation, such as living with a partner, with a child, or alone. Factor 5 was best described as Employment Situation, as the highest loading variables were whether a person was employed full time before the pandemic, was retired, and whether their employment during the pandemic changed to working from home. Finally, Factor 6 was described as City Type, as the three highest loadings were whether someone was living in a suburban, urban, or rural area (see Supplementary Material, Table A3.2).

In order to understand individual differences, the mean and standard deviation for the variables were computed for people whose factor scores were in the top $25 \%$ of all responses and people whose factor scores were in the bottom 25\%. The difference in means and standard deviations between the "high" and "low" scorers was then calculated. Due to the large number of variables (80) and the resulting risk of false positives, we elected not to perform any $t$-tests or statistical comparisons, as there would be a total of 1600 tests performed ( 80 variables $* 5$ factors * 2 conditions: music making and music listening $* 2$ scores: mean, standard deviation). Instead, we report the variables with the largest differences in means and standard deviations among the high and low scorers. The complete tables summarising these differences can be found in our GitHub repository (https://github. com/lkfink/CMQ). Although the cutoff marking high and low scorers (top 25\% and bottom 25\%) is arbitrary and no formal statistical analyses were conducted, high scorers and low scorers exhibit clearly distinct profiles on their wellbeing indices (see Fig. A3), which gives credit to this approach.

Statistical modelling. On the individual level, our goal was to predict participants' responses to the questions asking the degree to which they agreed that listening to music and making music during the lockdown (a) helped them cope emotionally with their present situation, (b) made them feel connected to others, and (c) served as a replacement for social interaction. We were interested in differences in using music listening and music making for socio-emotional coping and, therefore, conducted a separate analysis for each of these two domains.

Pre-processing included removing the emotional variable "Boredom" and all music listening formats (e.g., radio, streaming, etc.) because, due to an error, not all countries saw these questions. Missing values were again imputed with the median of the variable for that participant's country of residence. Because the three measures of socio-emotional coping were highly correlated (all Pearson $r$ values were between 0.65 and 0.72 ), the average of these three items was computed to serve as a holistic socio-emotional coping score for each participant. Participants who did not respond to all of the coping questions (a-c above) were removed from the analysis. This process was the same for music listening and music making analyses. All music making variables were ignored in the music listening model and vice versa (i.e., all listening variables were excluded as predictors in the music making model).

Linearity assumptions were tested with regards to both the music listening and making data separately. Linear regression models predicting the average coping scores resulted in eigenvalues very close to 0 (smallest eigenvalue for music listening $=7.53 \mathrm{e}-19, \quad$ smallest eigenvalue for music performance $=3.41 \mathrm{e}-18$ ), suggesting that there were strong multicollinearity problems in the two datasets or that the design matrices were singular. In addition, 24 variables in the music listening model (and 24 in the making music model) had VIF scores $>5$. In both datasets, the residuals were nonnormal and the Jarque-Bera test revealed that the data were not normally distributed (music listening: Jarque-Bera statistic $=270.535, \quad p<0.01, \quad$ skew $=-0.491, \quad$ kurtosis $=3.663$; making music: Jarque-Bera statistic $=450.022, \quad p<0.01$, skew $=-0.692$, kurtosis $=4.287$ ). Finally, Breush-Pagan tests indicated that both datasets violated the assumption of homoskedasticity (music listening: Lagrange multiplier statistic $=434.319, p<0.01, f$-value $=3.008, f-p<0.01 ;$ making music: Lagrange multiplier statistic $=256.417, \quad p<0.01$, $f$-value $=1.714, f-p<0.01)$. As such assumptions were violated, it was necessary to use a non-linear model.

The Light Gradient-Boosted Machine (LGBM) regressor model, first published in 2017, was selected, as its gradient boosting framework and leaf-wise growth were optimal for the 
current dataset and its high speed and low memory requirements exceed the capabilities of other tree-based models, like Random Forest regression (Ke et al., 2017). For the music listening data, the holistic coping score served as the dependent variable, while the demographic, COVID situation, activities, functions, situations, and selection data were used as the predictors. An 80-20 train-test split was used. A grid-search with a threefold crossvalidation was conducted on the training data in order to choose the optimal hyperparameters for the training set. The hyperparameter grid was standard and included learning rate, number of estimators, number of leaves, max depth, subsample for bin, and minimum child samples. The model fit on the training data that resulted in the best hyperparameters was then fit on the test set. The final music listening model was the following: LGBMRegressor(learning_rate $=0.05, \quad$ max_depth $=10, \quad$ min child_samples $=10, \quad$ num_leaves $=40, \quad$ random_state $=123$, subsample_for_bin $=500)$. The same process was conducted for the making music data. The final model parameters were: LGBMRegressor(learning_rate $=0.05, \quad$ max_depth $=10$, min_child_samples $=10$, num_leaves $=40$, random_state $=123$, subsample_for_bin $=1000)$. The code producing the LGBM regression can be found on our GitHub repository (https:// github.com/lkfink/CMQ).

Feature importances were assessed through the use of SHAP values (SHapley Additive exPlanations), which are the average of the marginal contributions across all permutations. SHAP values unify many existing methods of feature importance (LIME, Shapley sampling values, DeepLIFT, QII, Layer-wise relevance propagation, Shapley regression values, Tree interpreter) by using additive feature importance methods across these methods. SHAP values have also been shown to be more consistent with human intuition than other methods of determining feature importance (Lundberg and Lee, 2017).

\section{Results}

Regarding the impact of COVID-19 on participants' lives, in general, the individual samples showed large similarities with what could be expected for the general population in their countries (see Supplementary Material, Appendix 2). Across all countries, peoples' professional lives were most frequently affected by the crisis. According to official numbers, for example, 10.1 million (of $45 \mathrm{M}$ ) Germans (Bundesagentur für Arbeit, figures from April 26, 2020) and 11.3 M (of appr. $30 \mathrm{M}$ ) in France (La ministre du Travail, April 29, 2020) had to work reduced hours in April ("Chomage partiel"/"Kurzarbeit"), while 120 M Indians lost their job in April. In the US, unemployment rates went up from 4.4 to $14.7 \%$ in April, $2020 .^{3}$ Over half of our sample reported a change in their work situation, such as having to work reduced hours, suffering from a lower or no income, or seeing their job at risk. Various forms of known contact with the virus, however, were much less frequently reported, ranging from only $10 \%$ of Germans to $33 \%$ of those in the USA. Additional possible stressors included living alone (i.e., extreme social isolation, $15 \%$ of respondents) or having to home-school children $(31 \%$ of respondents reported living with child(ren) and $11 \%$ reported home-schooling their children). Further, reflecting the intense lockdown measures in place in most countries at the time of our data collection, participants reported leaving their house an average of only 3.8 times in the span of the past 7 days.

Additionally, participants' wellbeing changed during, compared to before, lockdown. Self-reports of negative emotions, stress, anxiety, loneliness, and depression all increased, on average, across countries, while positive emotions and energy decreased. However, often India was an exception, reporting more positive emotions and better general health than other countries. For full details, see Supplementary Material, Appendix 2. Nonetheless, collectively, the results are consistent with the idea that the majority of our participants were impacted by the COVID-19 shutdown and confinement, if not by the virus itself. As a consequence, the need to adapt and cope with confinement and anxieties about one's work situation were particularly urgent.

General changes in musical engagement during lockdown. Across all types of musical engagement polled, the range of participants reporting that they adapted their behaviours since confinement measures were introduced in their area was between 34 and 57\%. Changes were particularly pronounced for music selection behaviours (e.g., 57\% had developed moderate to extreme interest in coronamusic) and for the functions music making (56\%) and listening (51\%) should serve. From a list of 29 everyday domestic activities, music listening ranked directly behind critical ways of staying up-to-date with the outside world (e.g., calling people, watching news) and chores resulting from home confinement (e.g., cleaning, cooking; Fig. 1A). The only other non-necessary, leisurely behaviour ranking higher than music listening was watching movies or television series.

During the lockdown, people used music mainly to positively influence their individual emotional valence and arousal levels. The top ten functions that increased most in importance for listening (Fig. 1B) and making music (Supplementary Material, Fig. A4.1) all point towards mitigating negative states and emotions and boosting positive ones, most importantly via enjoyment in music itself ( $r a n k 1)$.

Changes in music listening situations, reported by $42 \%$ of participants, mirrored their confinement: Situations that ranked as more likely compared to before the crisis were forms of solitary and pure music listening, as well as music listening during housework or exercising. Conversely, music listening during social situations (e.g., while with others, dining, during sexual activity, etc.) became much less likely (see Supplemental, Fig. A4.2). On the other hand, $38 \%$ of people who made music reported that the situations in which they did so changed during the lockdown. The top music making situations included solitary forms (e.g., singing, dancing, and making music alone) and singing together via the internet (Supplemental, Fig. A4.3). It is interesting to note that, despite the broad media coverage, singing on balconies did not rank very highly on average in any of our surveyed countries, including Italy; in fact, its median rank across all countries was 10 (out of 13).

In contrast to the widespread interest in coronamusic, other changes in music selection behaviour were less pronounced. Only $34 \%$ of people reported listening to, making, or singing different music than before. Of these individuals, $31 \%$ turned to different musical genres/styles, compared to options that implied a lesser degree of novelty, such as listening to other pieces from the same musicians/composers (15\%) and listening to music by other musicians/composers but in a familiar genre/style (7\%). Thirtysix percent of participants reported an increase in listening to nostalgic music and $27 \%$ reported an increase in the diversity of music they listen to, play, or sing.

Individual differences in lockdown experience and associated musical behaviours. A factor analysis conducted on the demographic and personality variables resulted in six latent factors (see Supplementary Material, Appendix 3, for full factor loadings). One of the factors explained changes in negative emotions during the coronavirus crisis (with high loadings of depression, loneliness, stress, anxiety, and negative emotions), while another factor explained changes in positive emotions during the crisis (with high loadings of positive emotions, energetic arousal, and 
A

Domestic Activities

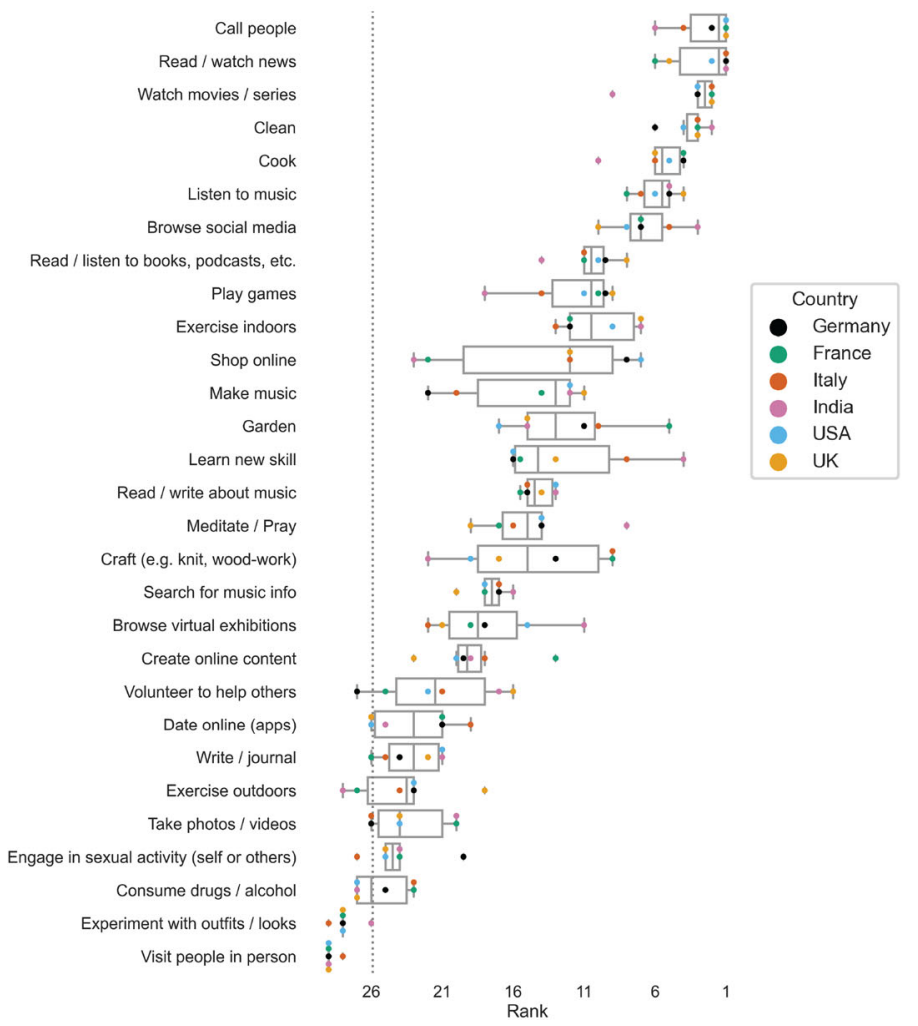

B

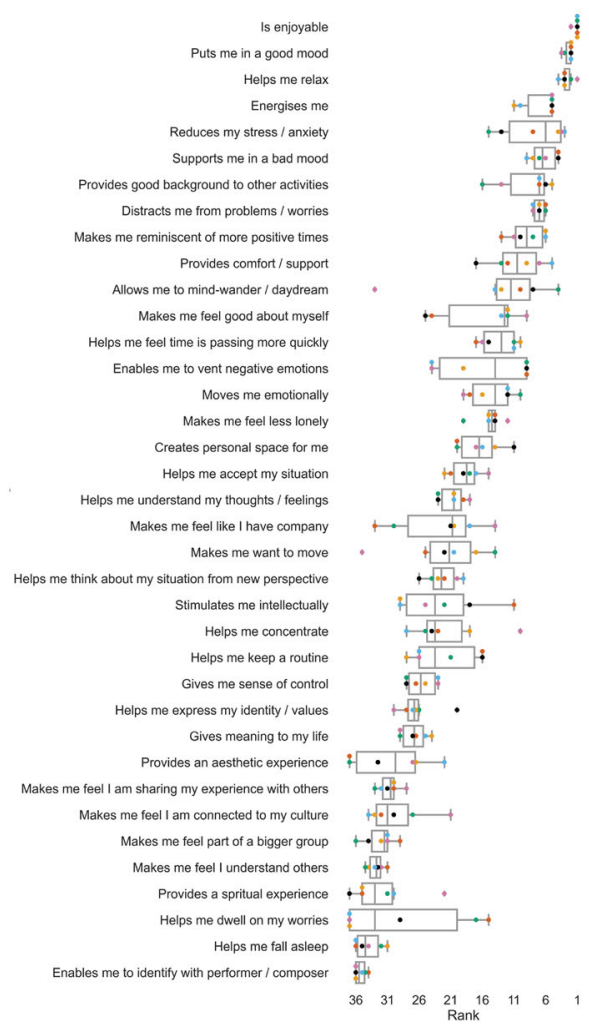

Fig. 1 Ranked domestic activities and functions music listening should serve. A Lockdown activities, $n=4206$, and B functions of listening to music, $n=4146$, ranked by mean change in importance of each item, within country. Final ranks are displayed according to median rank across all countries. The dotted vertical line in $\mathbf{A}$ represents the change point left of which items became less important, on average, across all countries. No vertical line is drawn in B because all items became more important, on average. Box plots represent the dispersion of rankings across countries, for each item. Each dot represents a country. The left and right sides of the boxes represent the 25th and 75th percentiles, with a line at the median. Error bars represent the lowest and highest ranks within a 1.5 interquartile range (IQR). Points falling outside the IQR are defined as outliers.

general health). The remaining factors described a person's age, living situation (alone or with others), employment status, and city type (urban, suburban, rural).

A descriptive analysis investigated differences in musical behaviours among people with various levels of change in experienced positive and negative emotions during the coronavirus crisis to understand whether behavioural changes can be interpreted as adaptive. A comparison of the $25 \%$ of participants who scored highest and $25 \%$ who scored lowest on the Positive Emotions and Negative Emotions latent factors revealed specific differences with regard to coping behaviours and changes in functions, situations, and music selection. On average, the top $25 \%$ on the Negative Emotions factor reported experiencing negative emotions more frequently during lockdown than before the pandemic, while the low $25 \%$ reported experiencing negative emotions less often (see Supplemental, Fig. A3 for details). In contrast, the top quartile on the Positive Emotions factor reported experiencing positive emotions more frequently, while the low quartile reported experiencing positive emotions less often (Supplemental, Fig. A3). These two emotion-related factors also included aspects of the pandemic and the lockdown, albeit with much smaller loadings (see Supplementary Material, Appendix 3). Both Positive and Negative Emotions factors had loadings of items that asked about participants' direct contact with the virus (range of loadings on negative emotions factor: $0.11-0.17$, on positive emotions factor: $0.12-0.31$ ). The Negative Emotions factor was weakly associated with the personality trait neuroticism (factor loading of 0.31 ), as well as an impairment in people's work situation (lost job, job at risk, reduced income; factor loadings $0.12-0.14$ ). The Positive Emotions factor was also weakly associated with working reduced hours, as well as living with a child and in an urban surrounding (factor loadings $0.12-0.13)$.

As detailed in Table 1, people who reported higher amounts of Negative Emotions during the pandemic utilised music listening and making mostly as a way to cope emotionally with the crisis and thus used it mostly to reduce negative affect, such as stress and loneliness, as well as to provide a sense of comfort and support. People whose Positive Emotions increased during the lockdown tended to experience music listening and making specifically as a form of social coping (as can be seen from the larger mean differences for the coping items "feel connected to others" and "serve as social interaction") and reported to use musical engagement not only to have some form of imaginary exchange, but also to have a special (aesthetic or spiritual) experience.

With regard to music listening situations, people with various levels of changing Negative Emotions differed mainly in situations in which they were alone or conducting another activity. Compared to people whose Positive Emotions decreased during the pandemic, people whose Positive Emotions increased reported listening to music more frequently together with others and in situations that would allow them to listen attentively (in the evening, while travelling). For music making situations, people whose Positive Emotions increased significantly during the lockdown tended to engage with various forms of dancing, 
Table 1 Overview of individual differences in music-related coping, functions, situations, and selection for two latent variables that describe changes in positive and negative emotion during the coronavirus crisis.

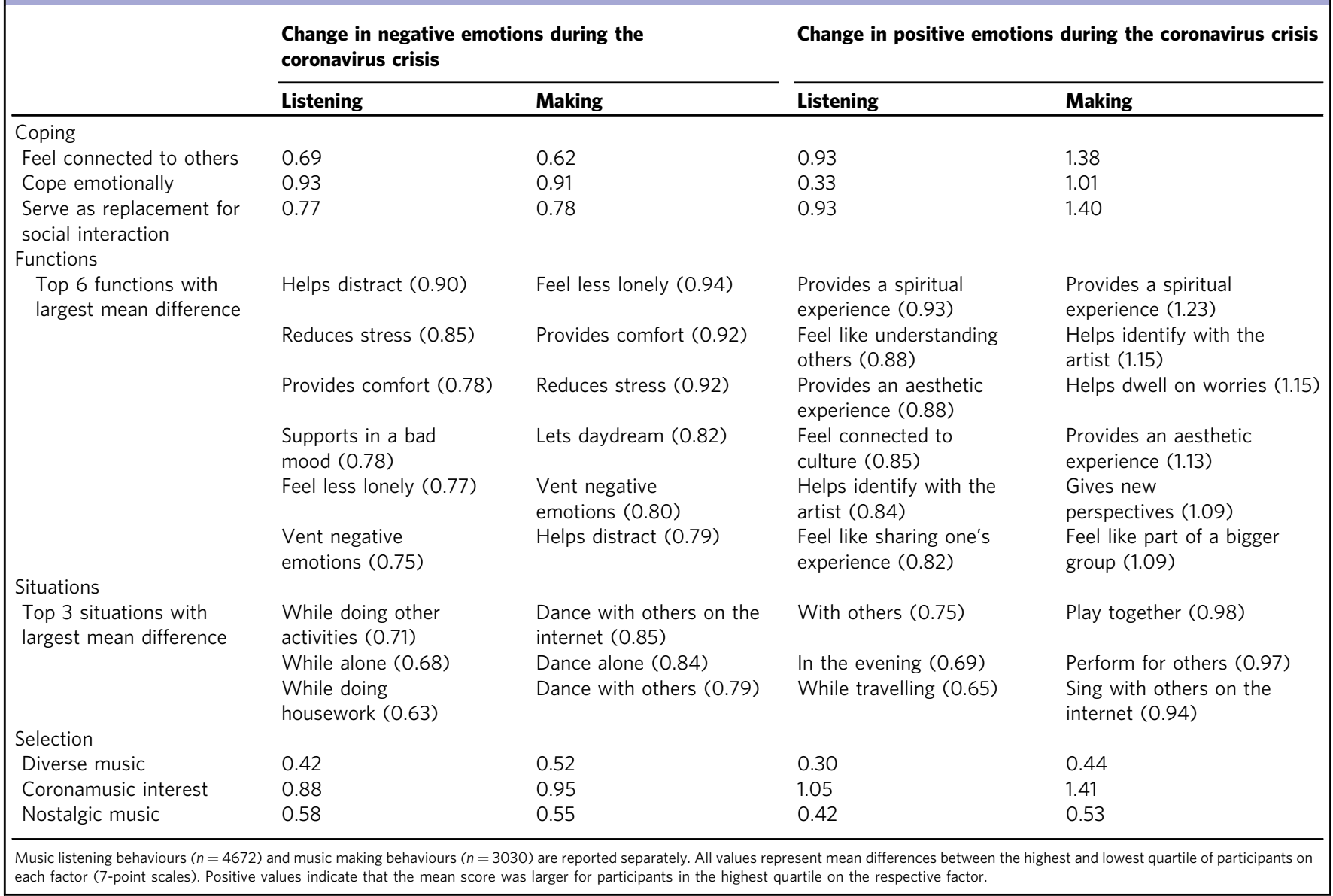

whereas people who experienced higher levels of Positive Emotions during the pandemic were more likely to perform with and for others. Both groups used forms of making music online.

Similar analyses comparing people in the highest and lowest 25th percentiles for the remaining factors did not result in any notable differences.

Variables predicting the use of music for socio-emotional coping. Socio-emotional coping was defined as the average of three questions, all of which had Pearson correlations between 0.65 and 0.72: music listening or music making "makes me feel connected to others," "serves as a replacement for social interaction," and "helps me to cope emotionally with the present situation." Across all countries, 55\% of respondents who reported listening to music ( $n=4626$ people) and $57 \%$ of those who made music $(n=3023)$ also used it to cope more often than before the lockdown (i.e., scored an average above 4 on the 7-point Likert scale for the three combined coping questions). Two light gradient-boosted machine (LGBM) regressor models were used to predict the degree of reported socio-emotional coping from all other variables (Fig. 2). The model predicting socio-emotional coping via music listening explained $53.6 \%$ of the variance in coping scores and the model predicting socio-emotional coping via making music explained $54.4 \%$ of the variance in coping scores. The low mean absolute errors in the two models indicate that the average error of the models was small (music listening: $\mathrm{MAE}=0.79$, making music: $\mathrm{MAE}=0.77$ ).
The majority of the 20 most important predictors in both LGBM models were music-related, of which most denoted a behavioural adaptation with regard to specific functions of music listening or making. Both models shared seven predictors: an interest in coronamusic and selecting different music than before the crisis (two facets of music selection behaviour), the social surrogacy functions "feel like having company" and "feel like being part of a group," the general importance of music to the individual, as well as the personality trait neuroticism and the confirmed number of COVID-19 infections.

Predictors for socio-emotional coping via music listening (Fig. 2A) included additional social surrogacy functions ("reduces loneliness," "feel like sharing one's experience") and a number of self-directed functions that were related to mitigating negative states ("creates a personal space," "provides comfort and support," "feel good about self," "gives new perspectives," "reduces stress"). Additionally, a change in the importance of music listening, and listening to music during other activities also predicted the degree to which people experienced music listening as helping them cope during the pandemic. The only non-musical predictors were personality traits (with lower levels of agreeableness, neuroticism, and extraversion being related to less coping via music listening), age (with older people reporting higher degrees of coping), and changes in anxiety level during lockdown.

Social surrogacy functions were less prominent as predictors with regards to using making music to cope during the coronavirus crisis (Fig. 2B). Instead, predictive functions centred mainly around creating positive states ("helps understand feelings," "puts in good 
A

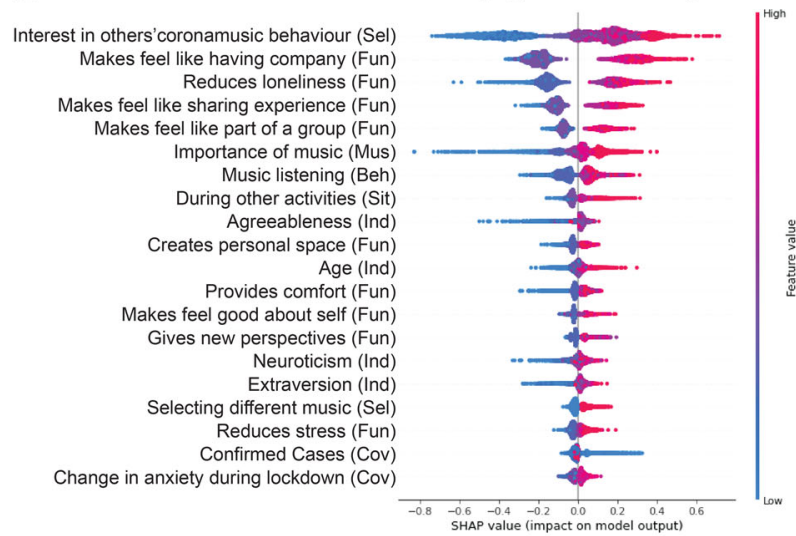

B

Predictors of socio-emotional coping via music making

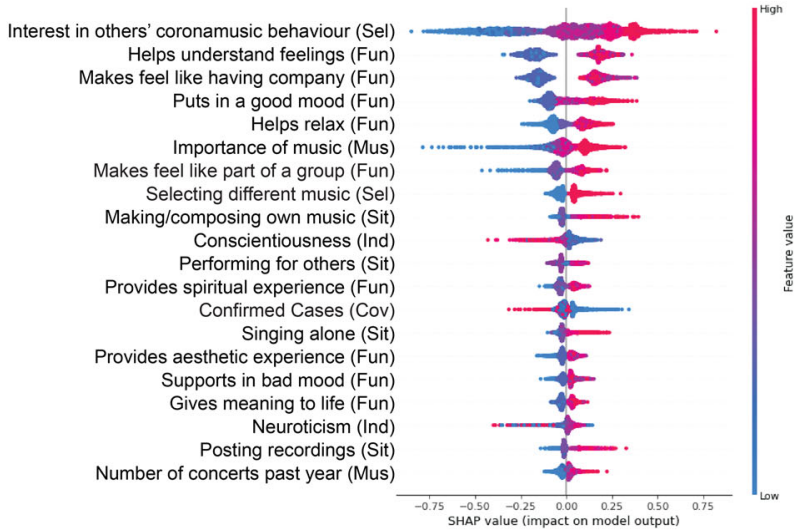

Fig. 2 Predictors of socio-emotional coping. Top 20 features predicting socio-emotional coping via (A) music listening, $n=4626$, and (B) making music, $n=3023$. Data points represent Shapley additive explanation (SHAP) values for every person on each of the top 20 most predictive features. SHAP values are measures of feature importance derived from game theory. The SHAP value for a given variable is a measure of the difference between two model predictions: one with the variable included and one without (Lundberg and Lee, 2017). Colour indicates whether a person scored high (red) or low (blue) on that variable. The $x$-axis shows the impact on the predicted socio-emotional coping score. Points to the right of 0 indicate that these feature scores increase the predicted socio-emotional coping score, whereas points to the left of the vertical black line decrease it. The greater the SHAP value (in absolute magnitude), the greater the impact on predicted socio-emotional coping score. Beh: Change in importance or frequency of behaviours during lockdown; Cov: COVID-19-related variable; Fun: Change in importance of functions of music listening/making during lockdown; Ind: individual characteristic (not music-related); Mus: music-related individual characteristic; Sel: Change in music selection during lockdown; Sit: Change in likelihood of situation of music listening/making.

mood," "helps relax") and meaningful experiences (spiritual and aesthetic experiences, "gives meaning to life"). Further, making music in situations that facilitated creative and communicative musical activities ("make own music," "perform for others," "post recordings of oneself on the internet") helped people cope. Active concertgoers (pre-lockdown) were also more likely to make music to cope. Finally, personality traits affected coping via making music, whereby higher degrees of conscientiousness and neuroticism predicted lower coping scores.

\section{Discussion}

Drawing on data from demographically representative samples in six countries from three continents, we showed that more than half of the surveyed adult population engaged with music as a strategy for coping with emotional and social stressors in the context of the first COVID-19 lockdown in early 2020 (mid-April through mid-May). By adopting a practice-based understanding of music that is also more suitable for cross-cultural research (Small, 1998; Turino, 2008), we showed that it was not the music itself that served as a coping aid, but the purposeful ways people engaged and interacted with it (i.e., their music-related behaviour). In particular, people who had been emotionally affected by the crisis were more likely to change their musical behaviours. Self-reported coping via listening to or making music was mainly predicted by changes in behavioural adaptations in the functions and situations of musical engagement, as well as in music selection behaviour. In particular, an interest in coronamusic was the strongest predictor of socio-emotional coping; demographic factors and more stable individual traits played a smaller role. Overall, our data suggest four general themes in the context of the coping potential of musical engagement, each to be discussed in turn.

During the COVID-19 lockdown, people have turned to music for regulating their emotions. The adult population engaged with music primarily to support emotion-focused coping during the COVID-19 lockdown. Music listening was one of the activities whose importance increased most during the COVID-19 lockdown compared to other leisure activities that could also facilitate emotion regulation, such as exercising, gardening, meditating, crafting, or using drugs. Of these leisure activities, music might also be more widely and easily accessible. From a range of nearly forty functions, those related to regulating negative and boosting positive affect increased the most in importance during the COVID-19 lockdown, such as enjoyment, relaxation, comfort, distraction, and a reduction of stress and anxiety. All of these functions directly target the negative wellbeing effects of the COVID-19 pandemic (Dagnino et al., 2020; Dawel et al., 2020; Franceschini et al., 2020; Groarke et al., 2020; Hur, et al., 2020; Lenzo et al., 2020; Lippold et al., 2020; Parlapani et al., 2020; Rajkumar, 2020; Rodríguez-Rey et al., 2020; Rossi et al., 2020). Ultimately, these findings extend our knowledge about music usage to a global public health crisis, beyond previous studies that have identified the importance of music as a tool for emotional regulation in everyday life (Juslin and Sloboda, 2010; Saarikallio, 2011; Thoma et al., 2012; Randall et al., 2014; Groarke and Hogan, 2016, 2019).

People experiencing different types of emotional changes showed different patterns of musical engagement. The urgent need for a coping strategy able to alleviate negative emotions was also attested by the large degrees of experienced changes in the frequency of negative and positive emotions participants reported, which, in turn, we found to be associated with differences in music-related behaviours. People who were experiencing more negative emotions during the lockdown engaged with music primarily to cope with depression, loneliness, anxiety, and stress. On the other hand, of those people whose positive emotions changed, it was those whose positive emotions increased during lockdown-rather than those whose positive emotions decreased -who changed their musical behaviours and felt music helped them cope. These individuals with increased positive emotions reported listening to and making music primarily to serve as a proxy for social interactions and to have meaningful experiences, compared to people with decreased levels of positive emotions. The difference between the use of music to cope with changing positive and negative emotions is striking. While most changes in music behaviours related to changing negative emotions are solitary, the musical behaviours associated with changing positive emotions are more social. 
Such results indicate that people who experience large increases in their negative emotions due to the coronavirus crisis (but not those who experience decreases in positive emotions) use music as a form of Solace. Solace is a personal and private regulation strategy which involves using music to feel accepted, understood, and comforted in times of sadness or trouble (Hanser et al., 2016; Saarikallio, 2008; Saarikallio and Erkkilä, 2007) and has been found to increase in frequency and importance during hardships and difficult life experiences (Hanser et al., 2016; Saarikallio, 2011). Previous work has shown that Solace is more adaptive than other regulation strategies used for dealing with negative states (such as expressing and releasing negative emotions) and may even act as a protective factor against mental health risks (Carlson et al., 2015). The lockdown severely reduced peoples' opportunities to rely on interpersonal support and left many to themselves. Thus, people put music in the role of a compassionate vis-à-vis, with Solace-related functions increasing in importance as people attempted to meet their own socio-emotional needs.

Music listening and music making may provide different coping potentials. The behavioural adaptations that predicted individuals experiencing music listening or music making as coping aid differed. The strongest predictors of coping via music listening included several functions related to the Solace regulation strategy, suggesting that, while these functions were subjectively important for both music listening and making, Solace was more effectively employed as a coping strategy through music listening. In contrast to listening, coping via music making was associated with generating positive affect, such as relaxation, good mood, and meaningful aesthetic and spiritual experiences-aspects that could be linked to regulation strategies such as revival, entertainment, and strong sensation (Saarikallio, 2008)-as well as with situations that involved creativity and communication with others.

Overall, our findings point to different roles for these two main musical behaviours (listening vs. making), which should be further substantiated by future research, especially given that previous studies have either not systematically looked into their potential difference or have found similar effects for both listening and making (Saarikallio, 2008). Our results can be seen as reflecting differences in the agency a person lends to the music and themselves in a situation of musical coping. Reducing negative emotions via listening implies an expectation that the music is the agent, turning to the listener and addressing them in their distress. Conversely, making music requires that people take action and see themselves as having agency. Here, the music is not so much a partner, but a means of self-expression, reflection, and a way to address others, and thus tends to merge with the performer. Therefore, in addition to the emotion regulation potential provided by listening, making music may also provide differential coping potential, such as perceived control over one's situation or enacting a sense of self-efficacy. Whether people resort to the one or the other might also depend on their personality, as evidenced by the differentiating roles that four of the five main personality traits played in predicting coping behaviour.

Coronamusic played a key role in socio-emotional coping. A surprising finding was that participants demonstrated a strong interest in corona-related musical repertoires and practices. This interest played a key role in predicting participants' use of music listening and/or making to cope with the lockdown situation. Such findings shed light on the importance of music selection and situation-specific musical content, two aspects which have only been sporadically touched upon in studies on musical coping and emotion regulation (Park et al., 2019; Van den Tol et al., 2016), though previous research has shown the relevance of functions and situations on music selection behaviour (Greb et al., 2018).

The crucial importance of the new coronamusic repertoire is also affirmed by the millions of clicks many such tracks or videos have received worldwide. Dedicated repertoires that give voice to a society's conflicts, crises and traumas have been created throughout history (e.g., in times of plague (Chiu, 2017) and wars (Mullen, 2019), or as protest (Friedman, 2013) or disaster songs). ${ }^{4}$ The attractiveness and coping potential of coronamusic may primarily lie in its characteristic as a medium that explicitly addresses the COVID-19 pandemic and its consequences on people's lives and feelings. Coronamusic covers a broad range of topics, with various emotional tones, in an often creative and aesthetically appealing way. In a sense, it can be seen as a return of "Umgangsmusik" (i.e., functional, communicative, vernacular music; Besseler, 1926/2011), or participatory music-making (Turino, 2008)-supposedly primordial forms of music-making that directly reflect the situation or challenge a group of people is facing, offering a joint way of responding to it.

Music with lyrics, in particular, can be attributed with a specifically large coping potential given that it may combine topicality and social surrogacy with music's ability to influence peoples' emotions and provide Solace. Engagement with coronamusic may thus constitute the musical equivalent of approach coping whereby media consumers dedicate time to content with direct relevance for navigating the psychological stressors at hand (Knobloch-Westerwick et al., 2009). Recent findings indeed show that engagement with especially eudaemonic media content concerned with existential questions of meaning and purpose in life helped consumers cope with anxiety during the pandemic lockdown (Eden et al., 2020). In addition, much of the coronamusic repertoire seeks to provide information about the virus and essential hygiene measures in an attractive and convincing way, calling for peoples' understanding and compliance. Empathy (Pfattheicher et al., 2020) and reliable information (Zickfeld et al., 2020) have been shown to increase adherence to containment measures. Thus, coronamusic not only provides empathy to its audience but can also be expected to elicit it and, ultimately, serve as a means to influence human behaviour, problem-solving, and adaptive coping.

Future research needs to systematically explore the textual, musical, and visual features and affordances of this repertoire, with an eye towards understanding what makes a viral (pun intended) tune, with a widespread global reach. Indeed, given the likelihood of additional pandemics and lockdown-like situations in the future, as well as the clear importance of music in boosting individual and collective morale, our findings on the tailored benefits of musical behaviours and dedicated repertoires in the context of global lockdowns stand in stark contrast to the fact that musicians are one of the occupational groups whose livelihood has been most strongly affected by the shutdown and distancing measures (Spiro et al., 2021). Acknowledging the important role of their coronamusic in helping people to cope with the lockdown situation and persevere, governments might take action by commissioning pandemic tunes, funding musical activities, and setting up systematic compensation schemes. In this way, a threatened profession, a large part of the population, and social stability at large may all benefit.

\section{Data availability}

The cleaned data required to reproduce all statistical analyses and figures in the paper are publicly available in our Github repository: https:/github.com/lkfink/CMQ. 


\section{Code availability}

All analyses are publicly available in our Github repository: https://github.com/lkfink/CMQ.

Received: 23 January 2021; Accepted: 29 June 2021; Published online: 26 July 2021

\section{Notes}

1 Watch the original video on YouTube here: https://www.youtube.com/ watch? $\mathrm{v}=$ BtulL3oArQw\&t=1s. Read an article about the dance challenge here: https:// www.billboard.com/articles/news/international/9329174/vietnam-coronavirus-psasong-best-tiktok-dance-videos.

2 One World Together at Home: https://www.globalcitizen.org/en/content/one-worldtogether-at-home-impact/. One Love Asia: https://www.onelove.asia/.

3 https://www.bls.gov/opub/ted/2020/unemployment-rate-rises-to-record-high-14point-7-percent-in-april-2020.htm.

4 Sparling H (2020) Coronavirus songs vs. disaster songs. Disaster Songs in Canada, http://disastersongs.ca/coronavirus-songs-vs-disaster-songs/.

\section{References}

Aalbers S, Fusar-Poli L, Bieleninik $Ł$ et al. (2017) The effect of music therapy on cognitive functions in patients with dementia: A systematic review and metaanalysis. Aging Ment Health 22(9):1103-1112. https://doi.org/10.1080/ 13607863.2017 .1348474

Barrett CB (2020) Actions now can curb food systems fallout from COVID-19. Nat Food 1:319-320. https://doi.org/10.1038/s43016-020-0085-y

Besseler H (1926) Grundfragen des musikalischen Hörens. Jahrbuch der Musikbibliothek Peters 32:35-52

Besseler H (2011) Fundamental issues of musical listening (M. Pritchard, Transl.). Twentieth-Century Music 8(1):49-70. https://doi.org/10.1017/ S1478572212000011

Best LA, Law MA, Roach S et al. (2020) The psychological impact of COVID-19 in Canada: Effects of social isolation during the initial response. Can Psychol/ Psychologie canadienne 62(1):143-154. https://doi.org/10.1037/cap0000254

Betsch C (2020) How behavioural science data helps mitigate the COVID-19 crisis. Nat Hum Behav 4(5):438-438. https://doi.org/10.1038/s41562-020-0866-1

Braun SC, Krause AE (2020) Freedom of choice: Examining music listening as a function of favorite music format. Psychomusicology 30(2):88-102. https:// doi.org/10.1037/pmu0000254

Carlson E, Saarikallio S, Toiviainen P et al. (2015) Maladaptive and adaptive emotion regulation through music: a behavioural and neuroimaging study of males and females. Front Hum Neurosci. https://doi.org/10.3389/ fnhum.2015.00466

Chiu R (2020) Functions of music making under lockdown: a trans-historical perspective across two pandemics. Front Psychol. https://doi.org/10.3389/ fpsyg.2020.616499

Chiu R (2017) Plague and music in the Renaissance. Cambridge University Press.

Cuypers K, Krokstad S, Holmen (2012) Patterns of receptive and creative cultural activities and their association with perceived health, anxiety, depression and satisfaction with life among adults: the HUNT study, Norway. J Epidemiol Community Health 66(8):69-703

Dagnino P, Anguita V, Cifuentes S (2020) Psychological Effects of Social Isolation due to Quarantine in Chile: an exploratory study. Front Psychiatry. https:// doi.org/10.3389/fpsyt.2020.591142

Dawel A, Shou Y, Smithson M (2020) The effect of COVID-19 on mental health and wellbeing in a representative sample of Australian adults. Front Psychiatry. https://doi.org/10.3389/fpsyt.2020.579985

DeNora T (2000) Music in everyday life. Cambridge University Press

Derrick JL, Gabriel S, Hugenberg K (2009) Social surrogacy: How favored television programs provide the experience of belonging. J Exp Soc Psychol 45 (2):352-362. https://doi.org/10.1016/j.jesp.2008.12.003

Eden AL, Johnson BK, Reinecke L et al. (2020) Media for coping during COVID-19 social distancing: Stress, anxiety, and psychological well-being. Front Psychol. https://doi.org/10.3389/fpsyg.2020.577639

Friedman R (ed.) (2013) The Routledge history of social protest in popular music. Routledge, New York, NY

Franceschini C, Musetti A, Zenesini C et al. (2020) Poor sleep quality and its consequences on mental health during the COVID-19 lockdown in Italy. Front Psychol. https://doi.org/10.3389/fpsyg.2020.574475

Gabriel S, Young AF (2011) Becoming a vampire without being bitten: the narrative collective-assimilation hypothesis. Psychol Sci 22(8):990-994. https:// doi.org/10.1177/0956797611415541
Garrido S, Baker FA, Davidson JW et al. (2015) Music and trauma: The relationship between music, personality, and coping style. Front Psychol. https:// doi.org/10.3389/fpsyg.2015.00977

Greb F, Steffens J, Schlotz W (2018) Understanding music-selection behaviour via statistical learning. Using the percentile-Lasso to identify the most important factors. Music Sci (Lond) 1:1-17. https://doi.org/10.1177/2059204318755950

Greb F, Schlotz W, Steffens J (2018) Personal and situational influences on the functions of music listening. Psychol Music 46(6):763-794. https://doi.org/ $10.1177 / 2059204318755950$

Groarke JM, Hogan MJ (2016) Enhancing wellbeing: an emerging model of the adaptive functions of music listening. Psychol Music 44(4):769-791. https:// doi.org/10.1177/0305735615591844

Groarke JM, Hogan MJ (2018) Development and psychometric evaluation of the adaptive functions of music listening scale. Front Psychol. https://doi.org/ 10.3389/fpsyg.2018.00516

Groarke JM, Hogan MJ (2019) Listening to self-chosen music regulates induced negative affect for both younger and older adults. PLoS ONE. https://doi.org/ 10.1371/journal.pone.0218017

Groarke JM, Berry E, Graham-Wisener L et al. (2020) Loneliness in the UK during the COVID-19 pandemic: cross-sectional results from the COVID-19 Psychological Wellbeing Study. PLoS ONE. https://doi.org/10.31234/osf.io/j2pce

Hale T, Angrist N, Goldszmidt R et al. (2021) A global panel database of pandemic policies (Oxford COVID-19 Government Response Tracker). Nat Hum Behav 5(4):529-538. https://doi.org/10.1038/s41562-021-01079-8

Hansen NC, Treider JMG, Swarbrick D et al. (2021) A crowd-sourced database of coronamusic: Documenting online making and sharing of music during the COVID-19 pandemic. Front Psychol. https://doi.org/10.3389/fpsyg.2021.684083

Hanser WE, ter Bogt TF, Van den Tol AJ et al. (2016) Consolation through music: a survey study. Music Sci 20(1):122-137. https://doi.org/10.1177/ 1029864915620264

Harzing AW (2006) Response styles in cross-national survey research: a 26-country study. Int J Cross Cult Manag 6(2):243-266. https://doi.org/10.1177/ 1470595806066332

Hays JN (2005) Epidemics and pandemics: Their impact on human history. ABCCLIO, Santa Barbara

Howlin C, Rooney B (2021) Cognitive agency in music interventions: increased perceived control of music predicts increased pain tolerance. Eur J Pain. https://doi.org/10.1002/ejp.1780

Hur J, Smith JF, DeYoung KA et al. (2020) Anxiety and the neurobiology of temporally uncertain threat anticipation. J Neurosci 40(41):7949-7964. https://doi.org/10.1101/2020.02.25.964734

Jetten J, Reicher S, Haslam A et al. (2020) 10 lessons for dealing with a pandemic. Psychologist 33:30-32

Juslin P, Sloboda J (eds.) (2010) Handbook of music and emotion: theory, research, applications. Oxford University Press, Oxford

Ke G, Meng Q, Finley T et al. (2017) LightGBM: A highly efficient gradient boosting decision tree. In: Guyon I, Luxburg UV, Bengio S et al. (eds.) Advances in Neural Information Processing Systems 30. Conference on Neural Information Processing Systems, Long Beach CA, 4-9 December 2017

Knobloch-Westerwick S, Hastall MR, Rossmann M (2009) Coping or escaping? Effects of life dissatisfaction on selective exposure. Commun Res 36(2):207-228

Kroska EB, Roche AI, Adamowicz JL et al. (2020) Psychological flexibility in the context of COVID-19 adversity: associations with distress. J Contextual Behav Sci 18:28-33. https://doi.org/10.1016/j.jcbs.2020.07.011

Ladinig O, Brooks C, Hansen NC (2019) Enjoying sad music: a test of the prolactin theory. Music Sci. https://doi.org/10.1177/1029864919890900

Langley M, Coutts L (2020) Singing away the coronavirus blues. Making music in a time of crisis reminds us we belong. Griffith News, March 26, https://news. griffith.edu.au/2020/03/26/singing-away-the-coronavirus-blues-makingmusic-in-a-time-of-crisis-reminds-us-we-belong/

Lenzo V, Quattropani MC, Musetti A (2020) Resilience contributes to low emotional impact of the COVID-19 outbreak among the general population in Italy. Front Psych. https://doi.org/10.3389/fpsyg.2020.576485

Lippold JV, Laske JI, Hogeterp SA et al. (2020). The role of personality, political attitudes and socio-demographic characteristics in explaining individual differences in fear of Coronavirus: A comparison over time and across countries. Front Psych. https://doi.org/10.3389/fpsyg.2020.552305

Lundberg SM, Lee SI (2017) A unified approach to interpreting model predictions. Adv Neural Inf Process Syst 4765-4774. (Curran Associates, Inc.) http:// papers.nips.cc/paper/7062-aunified-approach-to-interpreting-modelpredictions.pdf

Macdonald K (2020) The Kanneh-Mason family live-streamed a brilliant Beethoven concerto in lockdown. Available via Classic fm https://www.classicfm. com/artists/sheku-kanneh-mason/family-isata-beethoven-live-stream/ Accessed 25 Jun 2021

Mullen J (ed.) (2019) Popular song in the First World War. An international perspective. Routledge, New York, NY 
North A, Hargreaves D (eds.) (2008) The social and applied psychology of music. Oxford University Press, Oxford

Ollen JE (2006) A criterion-related validity test of selected indicators of musical sophistication using expert ratings. Dissertation, Ohio State University.

Park M, Thom J, Mennicken S, Cramer H, Macy M (2019) Global music streaming data reveal diurnal and seasonal patterns of affective preference. Nat Hum Beh 3(3):230-236

Parlapani E, Holeva V, Voitsidis P (2020) Psychological and behavioural responses to the COVID-19 pandemic in Greece. Front Psychol. https://doi.org/10.3389/ fpsyt.2020.00821

Pfattheicher S, Nockur L, Böhm R, Sassenrath C, Petersen MB (2020) The emotional path to action: Empathy promotes physical distancing and wearing of face masks during the COVID-19 pandemic. Psychol Sci 31(11):1363-1373

Polo SM (2020) A pandemic of violence? The impact of COVID-19 on conflict. Peace Economics, Peace Science and Public Policy, 26(3). https://doi.org/ 10.1515/peps-2020-0050

Prohaska A, Racimo F, Schork AJ et al. (2019) Human disease variation in the light of population genomics. Cell 177(1):115-131. https://doi.org/10.1186/14797364-5-4-220

Randall WM, Rickard NS, Vella-Brodrick DA (2014) Emotional outcomes of regulation strategies used during personal music listening: a mobile experience sampling study. Music Sci 18(3):275-291. https://doi.org/10.1177/ 1029864914536430

Randall WM, Rickard NS (2017) Reasons for personal music listening: a mobile experience sampling study of emotional outcomes. Psychol Music 45 (4):479-495. https://doi.org/10.1177/0305735616666939

Rajkumar RP (2020) COVID-19 and mental health: A review of the existing literature. Asian J Psychiatr 52. https://doi.org/10.1016/j.ajp.2020.102066

Rammstedt B, John OP (2007) Measuring personality in one minute or less: a 10item short version of the Big Five Inventory in English and German. J Res Pers 41:203-212. https://doi.org/10.1016/j.jrp.2006.02.001

Rodríguez-Rey R, Garrido-Hernansaiz H, Collado S (2020) Psychological impact and associated factors during the initial stage of the coronavirus (COVID-19) pandemic among the general population in Spain. Front Psychol. https://doi. org/10.2139/ssrn.3623949

Rossi A, Panzeri A, Pietrabissa G et al. (2020) The anxiety-buffer hypothesis in the time of COVID-19: When self-esteem protects from loneliness and fear for anxiety and depression. Front Psychol. https://doi.org/10.3389/ fpsyg.2020.02177

Saarikallio SH (2008) Music in mood regulation: Initial scale development. Music Sci 12(2):291-309. https://doi.org/10.1177/102986490801200206

Saarikallio SH (2011) Music as emotional self-regulation throughout adulthood. Psychol Music 39(3):307-327. https://doi.org/10.1177/0305735610374894

Saarikallio S, Erkkilä J (2007) The role of music in adolescents' mood regulation. Psychol Music 35(1):88-109. https://doi.org/10.1177/0305735607068889

Schäfer T (2016) The goals and effects of music listening and their relationship to the strength of music preference. PLoS ONE 11(3). https://doi.org/10.1371/ journal.pone.0151634

Schäfer K, Eerola T (2020) How listening to music and engagement with other media provide a sense of belonging: an exploratory study of social surrogacy. Psychol Music 48(2):232-251

Schäfer K, Saarikallio S, Eerola T (2020) Music may reduce loneliness and act as social surrogate for a friend: evidence from an experimental listening study. Music Sci (Lond) 3:1-16. https://doi.org/10.1177/2059204320935709

Schäfer T, Sedlmeier P (2009) From the functions of music to music preference. Psychol Music 37(3):279-300. https://doi.org/10.1177/0305735608097247

Small C (1998) Musicking: the meanings of performing and listening. Wesleyan University Press, Middletown CT

Sparling H (2017) 'Sad and solemn requiems': disaster songs and complicated grief in the aftermath of Nova Scotia mining disasters. In H. Dell \& H. M. Hickey (Eds), Singing Death 90-104. London, UK: Routledge.

Theorell T, Kreutz G (2012) Epidemiological studies of the relationship between musical experiences and public health. In: MacDonald R, Kreutz G, Mitchell L (eds.) Music, health, and wellbeing. Oxford University Press, 424-435

Spiro N, Perkins R, Kaye S et al. (2021). The effects of COVID-19 lockdown 1.0 on working patterns, income, and wellbeing among performing arts professionals in the United Kingdom (April-June 2020). Front Psychol. https://doi. org/10.3389/fpsyg.2020.594086

Tarr B, Launay J, Dunbar RI (2014) Music and social bonding:"self-other" merging and neurohormonal mechanisms. Front Psychol 5:1096

Taylor S, Landry CA, Paluszek MM, Fergus TA, McKay D, Asmundson GJ (2020) COVID stress syndrome: Concept, structure, and correlates. Depress Anxiety 37(8):706-714

Thoma MV, Ryf S, Mohiyeddini C et al. (2012) Emotion regulation through listening to music in everyday situations. Cogn Emot 26(3):550-560. https://doi. org/10.1080/02699931.2011.595390
Trehub SE (2003) The developmental origins of musicality. Nat Neurosci 6 (7):669-673. https://doi.org/10.1038/nn1084

Turino T (2008) Music as social life: The politics of participation. University of Chicago Press, Chicago

Van den Tol AJ, Edwards J, Heflick NA (2016) Sad music as a means for acceptance-based coping. Music Sci 20(1):68-83. https://doi.org/10.1177/ 1029864915627844

Villa S, Jaramillo E, Mangioni D et al. (2020) Stigma at the time of the COVID-19 pandemic. Clin Microbiol Infect 26(11):1450-1452. https://doi.org/10.1016/j. cmi.2020.08.001

Warrenburg LA (2020) People experience different emotions from melancholic and grieving music. Music Sci (Lond) 3:1-23. https://doi.org/10.1177/ 2059204320977384

de Witte M, Spruit A, van Hooren S et al. (2020) Effects of music intervention on stress-related outcomes: a systematic review and two meta-analyses. Health Psychol Rev 14(2):294-324. https://doi.org/10.1080/17437199.2019. 1627897

Zickfeld JH, Schubert TW, Herting AK, Grahe J, Faasse K (2020) Correlates of health-protective behavior during the initial days of the COVID-19 outbreak in Norway. Front Psychology 11

\section{Acknowledgements}

This work was partially supported by a grant from the Irish Research Council to $\mathrm{CH}$ grant No. GOIPG/2017/1006. NCH received funding from the European Union's Horizon 2020 research and innovation programme under the Marie Skłodowska-Curie grant agreement No. 754513, Aarhus University Research Foundation, Lundbeck Foundation (R266-20173339), Carlsberg Foundation (CF18-0668), and a seed funding grant from Interacting Minds Centre (2020-153). The authors thank Rainer Polak and Pauline Larrouy-Maestri, MPI for Empirical Aesthetics, for their help with the German and French translations of the questionnaire, and Brendan Rooney from University College Dublin for facilitating ethics registration, and data management.

\section{Author contributions}

All authors contributed to study conceptualisation, design, and manuscript writing/ editing. LF and LW conducted all data analyses.

\section{Funding}

This work was partially supported by a grant from the Irish Research Council to $\mathrm{CH}$ grant No. GOIPG/2017/1006. NCH received funding from the European Union's Horizon 2020 research and innovation programme under the Marie Skłodowska-Curie grant agreement No. 754513, Aarhus University Research Foundation, Lundbeck Foundation (R266-2017 3339), Carlsberg Foundation (CF18-0668), and a seed funding grant from Interacting Minds Centre (2020-153). Open Access funding enabled and organized by Projekt DEAL.

\section{Competing interests}

The authors declare no competing interests.

\section{Additional information}

Supplementary information The online version contains supplementary material available at https://doi.org/10.1057/s41599-021-00858-y.

Correspondence and requests for materials should be addressed to L.F. or L.W.

Reprints and permission information is available at http://www.nature.com/reprints

Publisher's note Springer Nature remains neutral with regard to jurisdictional claims in published maps and institutional affiliations.

cc (i) Open Access This article is licensed under a Creative Common Attribution 4.0 International License, which permits use, sharing, adaptation, distribution and reproduction in any medium or format, as long as you give appropriate credit to the original author(s) and the source, provide a link to the Creative Commons license, and indicate if changes were made. The images or other third party material in this article are included in the article's Creative Commons license, unless indicated otherwise in a credit line to the material. If material is not included in the article's Creative Commons license and your intended use is not permitted by statutory regulation or exceeds the permitted use, you will need to obtain permission directly from the copyright holder. To view a copy of this license, visit http://creativecommons.org/ licenses/by/4.0/.

(c) The Author(s) 2021 